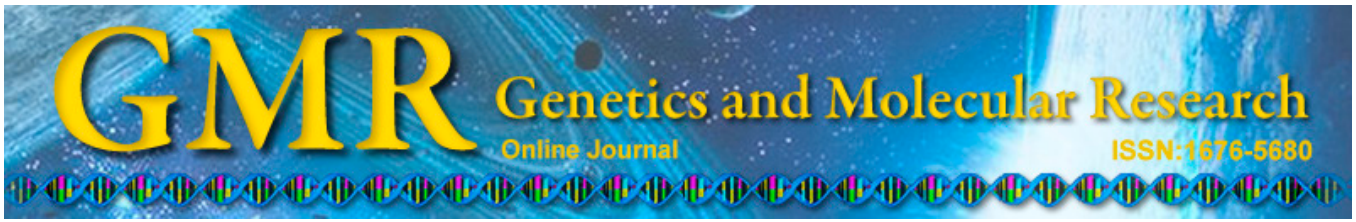

\title{
Effect of phosphatidylserine on memory in patients and rats with Alzheimer's disease
}

\author{
Y.Y. Zhang, L.Q. Yang and L.M. Guo \\ The Nursing School, Qiqihar Medical University, Qiqihar, China \\ Corresponding author: Y.Y. Zhang \\ E-mail: yingyanzhang@126.com
}

Genet. Mol. Res. 14 (3): 9325-9333 (2015)

Received January 7, 2015

Accepted May 8, 2015

Published August 10, 2015

DOI http://dx.doi.org/10.4238/2015.August.10.13

\begin{abstract}
The aim of this study was to investigate the effect of phosphatidylserine (PS) on memory of patients and rats with Alzheimer's disease (AD). In total, 57 AD patients were recruited from our hospital, and were divided into two groups: 25 in the control group and 32 in the observation group. Next, $300 \mathrm{mg} / \mathrm{d}$ of PS was given to the rats in the observation group for 12 continuous weeks based on the control group. AD rats were divided into three groups: control group, PS $30 \mathrm{mg} / \mathrm{kg}$ group, and PS $15 \mathrm{mg} / \mathrm{kg}$ group. Learning memory ability and free radical levels in the brain were detected after treatment. In AD patients, vocabulary and picture matching scores in the two treatment groups increased after treatment $(\mathrm{P}<0.05)$. Moreover, the scores in the treated group were significantly greater than the control group ( $\mathrm{P}$ $<0.05)$. In AD rats, PS treatment reduced the escape latent period of $\mathrm{AD}$ rats, increased SOD and $\mathrm{OH}^{-}$, and decreased acetylcholinesterase levels $(\mathrm{P}<0.05)$. Compared with PS $15 \mathrm{mg} / \mathrm{kg}$, PS $30 \mathrm{mg} / \mathrm{kg}$ group was significantly more efficacious $(\mathrm{P}<0.05)$. Compared with the AD model group, hippocampal cells showed normal arrangement, karyopyknosis decreased, and the pathological changes in the two PS groups were considerable. In conclusion, PS decreased cholinesterase, improved memory, and improved hippocampal inflammation injury in AD brains by increasing SOD and $\mathrm{OH}^{-}$levels.
\end{abstract}

Key words: Phosphatidylserine; Alzheimer's disease; Memory 


\section{INTRODUCTION}

Alzheimer's disease (AD) is the most common progressive neurological disease in elderly patients. The early clinical manifestations include impaired memory, disorientation, understanding difficulties, and analysis and calculation function decline. Generally, these patients can look after themselves in their daily lives. However, patients experience memory and analysis obstacles during middle and advanced periods, and the most severe suffers cannot look after themselves, which puts a tremendous burden on the patients, their families, and society. Therefore, AD has become a disease that seriously threats the lives and health of the elderly second only to cardiovascular and cerebrovascular diseases, and cancer (Ballard et al., 2011; Izzo et al., 2014). This aging problem has become increasingly prominent in our country at present, and the incidence of $\mathrm{AD}$ is increasing year by year. Treatments to delay or improve clinical symptoms of $\mathrm{AD}$ are therefore particularly urgent.

There is no specific drug for $\mathrm{AD}$; drugs to enhance cholinergic neurotransmitters is only used for maintenance at present, and their efficacy is unsatisfactory (Cagnin et al., 2014; Hou et al., 2014). Phosphatidylserine (PS) was extracted from bovine brains and quantified by Jordi Folch for the first time in 1942. It exists in nerve cells in the brain. PS can not only nourish the brain, but can also enhance brain function, relieve mental pressure, and improve cognition, memory, and reaction force. Therefore, it is also known as a "brain nutrient". In 1986, Delwaide first reported that bovine brain derived PS $(300 \mathrm{mg} / \mathrm{d})$ was orally administered to AD patients. Their results showed that PS could significantly improve cognitive function. A large number of European and American studies have also confirmed the efficacy of PS (Nair et al., 2014; Meng et al., 2014). However, there are few domestic studies on PS for AD. Therefore, in this study, the effect of PS on memory of $\mathrm{AD}$ patients was investigated, an $\mathrm{AD}$ rat model was established, and the possible mechanisms underlying the effect of PS on memory improvement in AD patients was studied.

\section{MATERIAL AND METHODS}

\section{Subjects}

In total, 57 patients admitted to our hospital and diagnosed with AD were recruited between September 2010 and January 2014. Exclusion criteria were as follows: vascular dementia, senile depression, hysteria or insane; severe heart, liver and kidney dysfunction; abnormal eyesight, failing to complete simple cognitive tasks or simple instructions; without treatment and incomplete data. The subjects were randomly divided into two groups: 25 in the control group and 32 in the observation group. All patients provided, signed informed consent. There was no significant difference in the general characteristics of the two groups (Table 1). This study was conducted in accordance with the Declaration of Helsinki and with approval from the Ethics Committee of Qiqihar Medical University.

\section{Treatment method}

All patients were given conventional $\mathrm{AD}$ treatment. Blood pressure and blood glucose were controlled. PS soft capsules (Nuotianyuan Trade Co., Ltd., batch 20100101, size 300 $\mathrm{mg} /$ particles) were administered orally in the observation group (300 $\mathrm{mg}$ once a day for 20 continuous weeks). 


\begin{tabular}{|c|c|c|c|c|c|}
\hline Items & & Control group & Observation group & $t / \chi^{2}$ & $\mathrm{P}$ \\
\hline Cases & & 25 & 32 & - & - \\
\hline Male:Female & & $12: 13$ & $16: 16$ & 0.0 & $>0.05$ \\
\hline Age & & $75.3 \pm 11.8$ & $74.9 \pm 18.2$ & 0.1 & $>0.05$ \\
\hline BMI $\left(\mathrm{kg} / \mathrm{m}^{2}\right)$ & & $20.1 \pm 0.8$ & $20.4 \pm 1.1$ & 1.1 & $>0.05$ \\
\hline Course of disease (years) & & $5.4 \pm 3.3$ & $6.0 \pm 4.5$ & 0.6 & $>0.05$ \\
\hline \multirow[t]{4}{*}{ Complications } & Hypertension & 12 & 15 & 0.0 & $>0.05$ \\
\hline & Coronary disease & 8 & 10 & 0.0 & $>0.05$ \\
\hline & Diabetes mellitus & 5 & 8 & 0.2 & $>0.05$ \\
\hline & Others & 3 & 3 & 0.1 & $>0.05$ \\
\hline \multirow[t]{2}{*}{ Level of education } & $\leq$ junior middle school & 15 & 14 & 1.5 & $>0.05$ \\
\hline & $\geq$ senior middle school & 10 & 18 & & \\
\hline \multirow[t]{2}{*}{ Degree of dementia } & Mild & 8 & 5 & 2.3 & $>0.05$ \\
\hline & Moderate & 14 & 21 & & \\
\hline
\end{tabular}

\section{Index detection}

Patient memory was investigated by semantic memory test before and after treatment. The semantic memory test is the relevant matching test developed in accordance with Chinese culture and the Beijing Normal University Cognitive Neuroscience and Learning Institute. It is composed of a combined set of 70 pictures and words. The content is derived from seven categories including animals, tools, vegetables, fruits, operable/inoperable objects, faces, and movements. Ratings were either 0 or 1 . The time for each question was limited to within $1 \mathrm{~min}$, and a higher score was suggestive of stronger recall ability.

\section{Experimental animals}

In total, 70 elder SPF level Wistar rats, 35 males and 35 females, weighing 500-550 $\mathrm{g}$, were purchased from the experimental animal center at Harbin Medical University. The animal Certificate No. was SCXK (black) 2006-010. Housing temperature was $24^{\circ}-28^{\circ} \mathrm{C}$ and the relative humidity was $45-65 \%$. The animals were housed in 12-h light/dark cycles. The rats were given adaptive feeding for 7 days before the experiment. This study was performed in strict accordance with the recommendations in the Guide for the Care and Use of Laboratory Animals of the National Institutes of Health, 8th Edition, 2010 (Bethesda, MD, USA). The animal use protocol was reviewed and approved by the Institutional Animal Care and Use Committee (IACUC) of Qiqihar Medical University.

\section{AD modeling and grouping}

Overall, 64 rats with memory conformity were screened using the MT-200 type Morris water maze experiment (Taimeng Technology Co., Ltd., Chengdu, China). First, the rats were anesthetized by chloral hydrate, placed in a stereotaxic apparatus, and fixed. Next, their skin was disinfected and incised. A craniotomy was performed $3.30 \mathrm{~mm}$ behind the anterior fontanelle incisor hook plane and $3.30 \mathrm{~mm}$ under the interaural line plane, and the cerebral dura mater was exposed. A needle was then inserted $3.0 \mathrm{~mm}$ into the subdural space with a microsyringe, namely in the bilateral hippocampi. Next, $5.0 \mu \mathrm{L} \alpha \beta 1-42$ solution was slowly 
injected into each side of the rats in each group with a speed of $1.0 \mu \mathrm{L} / \mathrm{min}$. After injection, the needle hole was filled with denture powder and the incision was sutured. Five drops of gentamicin was dripped into the incision $(30 \mathrm{mg} / \mathrm{kg})$ to prevent infection. After the cerebral dura mater was exposed in the control group, disinfection and suturing was performed, and gentamicin was injected. AD rats were randomly divided into three groups (16 rats/group; 8 male and 8 female rats). The treatment dose in the PS high dose group was 30, and $15 \mathrm{mg} / \mathrm{kg}$ in the PS low dose group. The rats in the model group and the control group were given distilled water for 30 continuous days instead of PS.

\section{Detection of learning memory}

Positioning navigation memory learning ability was determined in rats of each group by using the Morris water maze test. Briefly, the rats were placed into water from the second, third, and fourth quadrants of place of entry facing the pool wall. The time it took for the rat to find the platform within 2 min was recorded. The testing lasted for 4 days.

\section{Levels of free radicals in the brain}

After the last treatment, half of the rats were euthanized; the heads were broken on a glacial table and their brains were removed. Next, $10 \%$ brain tissue homogenate was prepared. SOD activity, hydroxyl radical inhibition rate, and cholinesterase levels were detected; the other half of the rats underwent immunohistochemistry detection. The brain was removed and fixed in paraformaldehyde, embedded in paraffin, stained, and observed under a microscope (Olympus, Tokyo, Japan).

\section{Statistical analysis}

All rat and patient data were input in the SPSS13.0 software package (SPSS Inc., Chicago, IL, USA). Data that conformed with normal distribution are reported as means \pm standard deviation. Comparisons between groups were performed using analysis of variance, and $\alpha=0.05$ was used as the test level. $\mathrm{P}<0.05$ was considered to be statistically significant.

\section{RESULTS}

\section{Comparison of memory test scores between the two groups}

There was no significant difference in vocabulary-picture matching scores between the two groups before treatment $(\mathrm{P}>0.05)$. Vocabulary-picture matching scores in two groups significantly increased after treatment compared to before treatment $(\mathrm{P}<0.05)$. Compared with the control group, scores were significantly higher in the observation group $(\mathrm{P}<0.05$; Table 2).

\section{Effect of PS on the escape latency of AD rats induced by $A \beta_{1-42}$}

During the experiment, the rats were generally in good condition in the control group, 
while those in the model group were dispirited, moved slowly, ate less, and experienced weight gain. Compared with control group, the escape latency in the AD model group was obviously prolonged, SOD and $\mathrm{OH}^{-}$were significantly decreased $(\mathrm{P}<0.05)$, and cholinesterase was significantly increased $(\mathrm{P}<0.05)$, suggesting that the $\mathrm{AD}$ model was successful. PS treatment significantly decreased the escape latency of $\mathrm{AD}$ rats, increased $\mathrm{SOD}$ and $\mathrm{OH}^{-}$, and decreased acetylcholinesterase $(\mathrm{P}<0.05)$. Compared with the low dose group, high doses significantly reduced the escape latency time, increased SOD and $\mathrm{OH}^{-}$, and decreased acetylcholinesterase $(\mathrm{P}<0.05$; Table 3$)$.

Table 2. Comparison of memory test score in two groups.
\begin{tabular}{lccc}
\hline Groups & Cases & Vocabulary matching score $\left(\times 10^{-1}\right)$ & Picture matching score $\left(\mathrm{x} 10^{-1}\right)$ \\
\hline Before treatment & & $8.8 \pm 0.8$ & $8.9 \pm 0.7$ \\
$\quad$ Control group & 25 & $8.7 \pm 0.5$ & $8.8 \pm 0.6$ \\
Observation group & 32 & 0.6 & 0.6 \\
$t$ & & $>0.05$ & $>0.05$ \\
P & & & $9.3 \pm 0.5^{*}$ \\
After treatment & 25 & $9.3 \pm 0.9^{*}$ & $9.7 \pm 0.7^{*}$ \\
Control group & 32 & $9.7 \pm 0.5^{*}$ & 2.4 \\
Observation group & & 2.1 & $<0.05$ \\
$t$ & & $<0.05$ & \\
P & & & \\
\hline
\end{tabular}

Table 3. Effect of PS on the escape latency, SOD and $\mathrm{OH}^{-}$of $\mathrm{AD}$ rats induced by $\mathrm{A} \beta_{1-42}(\mathrm{~N}=15)$.

\begin{tabular}{|c|c|c|c|c|c|}
\hline Groups & Dose & Escape latency & SOD (U/mg prot) & $\mathrm{OH}^{-}$(U/mg prot) & Cholinesterase \\
\hline Cases & & 16 & 8 & 8 & 8 \\
\hline Control group & - & $12.6 \pm 7.2$ & $105.2 \pm 16.2$ & $71.5 \pm 14.7$ & $16.8 \pm 5.7$ \\
\hline AD model group & - & $89.7 \pm 15.6^{*}$ & $53.4 \pm 9.7^{*}$ & $28.4 \pm 5.7^{*}$ & $69.7 \pm 11.4 *$ \\
\hline PS low dose group & 15 & $52.4 \pm 18.4^{\&}$ & $75.6 \pm 14.8^{\&}$ & $45.8 \pm 10.4^{\&}$ & $42.1 \pm 17.6^{8}$ \\
\hline PS high dose group & 30 & $21.1 \pm 5.6^{\& \#}$ & $92.4 \pm 11.3^{\& \#}$ & $58.2 \pm 13.3^{\& \#}$ & $29.7 \pm 8.7^{\& \#}$ \\
\hline$F$ & & 117.7 & 22.8 & 20.2 & 59.6 \\
\hline $\mathrm{P}$ & & $<0.05$ & $<0.05$ & $<0.05$ & $<0.05$ \\
\hline
\end{tabular}

Model group compared with control group, $* \mathrm{P}<0.05$. PS group compared with the model group, ${ }^{\&} \mathrm{P}<0.05$. Compared with low dose group, ${ }^{\mathrm{P}}<0.05$.

\section{Effect of PS on pathological tissue in AD rats}

Compared with the control group, hippocampal cells were disordered and a large number of karyopyknosis were evident in the model group. Conversely, hippocampal cells were arranged in order and karyopyknosis was significantly reduced in the PS group. These pathological changes were equivalent in the high and low PS dose groups (Figure 1).

\section{DISCUSSION}

The $\mathrm{AD}$ prevalence of residents over 60 years in domestic communities is more than $20 \%$, and the fatality ratio of $\mathrm{AD}$ is $47 \%$ in the United States each year. According to epidemiological predictions, one new case of $\mathrm{AD}$ will be diagnosed every $30 \mathrm{~s}$ in 2050 . Thus, the occurrence of or death from AD has become a social problem (Cong and Jia, 2011; Penolazzi et al., 2014). 
A

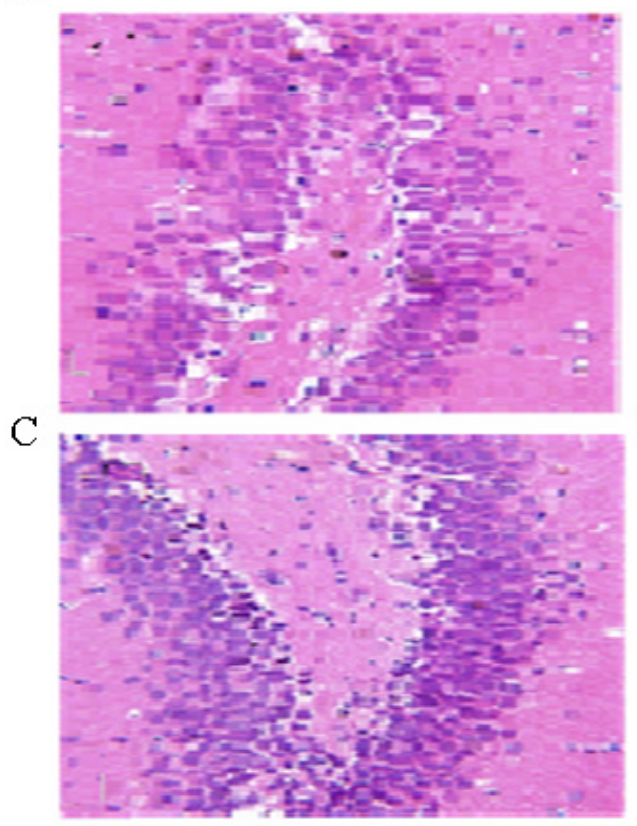

B

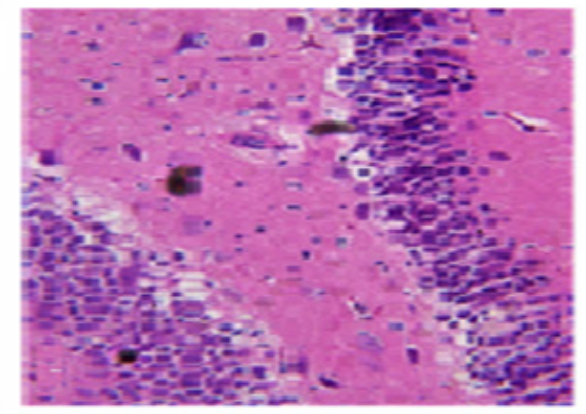

$\mathrm{D}$

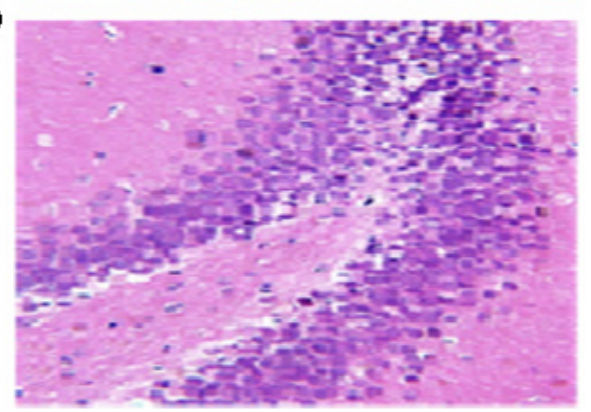

Figure 1. Effect of PS on pathological tissue of DA rats (Magnification 200X). A. Control group. B. Model group. C. PS low dose group. D. PS high dose group.

PS is an important substance in brain cell membranes, and is involved in brain cell membrane fluidity, and transmission of brain cell activity and neural information. With increasing of age, PS levels gradually decrease, thereby affecting memory and cognitive ability. PS supplement significantly enhances brain cell membrane fluidity, promote glucose metabolism in brain cells, and improves the physiological activities of protein kinase $\mathrm{C}$, acetylcholinesterase, tyrosine hydroxylase, and $\mathrm{Na}^{+}-\mathrm{K}^{+}$-ATP enzymes in the brain, and maintains the normal information transmission between nerve cells. Thus, brain cells are more active and brain structural deficiency due to brain fatigue and malnutrition were prevented with PS treatment (Manor et al., 2012; Hirayama et al., 2014; Schmid et al., 2014). The present results showed that AD patients taking $300 \mathrm{mg}$ PS/day for 20 continuous weeks significantly improved vocabulary and picture scores, which is consistent with clinical trial results showing that taking PS can improve memory in many healthy or memory impaired subjects. Dr. Crook from the Memory Assessment Clinics performed a cooperation development trial with 149 healthy subjects. The results showed that, compared with the placebo group, the daily use of PS for 12 weeks significantly improved the ability to remember names and faces, recalling names and faces, and recognizing faces. Among these patients, 57 with severe memory barriers (average age 64.3 years) took PS orally, remembering their phone number, recalling misplaced objects, attention during reading, talking, and working improved significantly. Zanotta et al. (2014) performed a double-blind test on 170 volunteers whose average age was 65.7 years old and who took $300 \mathrm{mg}$ PS/day for 3 continuous months. The results showed that, compared with the placebo group, attention and vigilance test results significantly improved. A long-term 
study showed that 425 subjects over 65 years of age with moderate to severe cognitive function degradation took $200 \mathrm{mg}$ /day of PS for continuous 6 months and processing information, recalling digital images, and emotion significantly improved. Finally, a diet containing PS for 60 continuous days significantly improved the cognitive scores of AD patients (Sato et al., 2010; Grimm et al., 2013; Zanotta et al., 2014).

PS has been studied substantially with respect to its impact on intelligence; however, its mechanism of action is unclear. Studies have shown that $A \beta$ aggregation is one of the underlying causes of $\mathrm{AD}$ (Canale et al., 2013). In the present study, $\mathrm{A} \beta$ was administered via intracerebral injection to rats to induce AD. Our results show that the escape latency of $\mathrm{AD}$ rats in the water maze test was significantly prolonged following this treatment. The rat brain can generate a large amount of oxygen free radicals, which induce nerve cell degeneration and central nervous system inflammatory responses. It is clear that $\mathrm{AD}$ development is related to peroxidation of brain cells damaged by free radicals, obvious inflammatory reaction, and increased acetylcholine, which is consistent with the clinicopathological features of AD patients. AD rats given PS (15 or $30 \mathrm{mg} / \mathrm{kg}$ ) showed significantly decreased escape latency in the Morris water maze and increased levels of SOD in the brain and improve hippocampal area pathology, which is consistent with results in the literature (Liu et al., 2012). Therefore, PS has an antioxidant function. Clinical trials have shown that PS can decrease the increase in mitochondrial membrane permeability in AD patients caused by non-steroidal anti-inflammatory drugs (Maiese and Chong, 2004), which further demonstrates the antioxidant efficacy of PS in animals and patients with $\mathrm{AD}$. In $\mathrm{AD}$ patients, the brain cholinergic system is damaged and the level of synaptic acetylcholine is reduced. Acetylcholine is closely related to human learning and memory function, and AD rats given PS showed decreased levels of cholinesterase in the brain. Our in vitro experiment showed that PS significantly increased acetylcholine levels in the brain. Cholinesterase can hydrolyze acetylcholine into choline and acetic acid thereby reducing acetylcholine levels. Thus, it is clear that PS increases the level of acetylcholine in the brain by inhibiting acetylcholinesterase. Although acetylcholine is a neurotransmitter, it cannot be synthesized by nervous tissue.

The predecessor of acetylcholine is choline. Choline is derived from food and is transported to neurons by blood. Choline can then be translated into acetylcholine for utilization in the brain. As a supplement, PS entering into cholinergic neurons can decarboxylate into phospholipids ethanolamine (PE). PE is then methylated to form phosphatidylcholine (PC). Wollen (2010) proved that PS protected neuronal cells by inhibiting A $\beta$ and inflammation (Wollen, 2010).

The limitations of this study include the fact that the source of PS used in our study included both animal brain and soybeans. It is controversial whether the pharmacological efficacy and safety of the two sources of PS are consistent. Richter et al. (2013) proposed that PS extracted from the bovine cerebral cortex can be added for an extended period of time, which has the obvious advantage of increasing the cognitive function of AD patients, and its safety is guaranteed. Suzuki et al. (2001) reported that PS derived from soybeans improve cognitive function, and can be used in the market. Richter et al. (2013) also reported that PS derived from soybeans can be used as a supplement to PS obtained from the bovine brain. However, the safety of PS derived from soybeans remains unknown. Wollen (2010) mentioned in his review that a reliable conclusion as to whether a PS derived from soybeans improved cognitive function tests without a positive control was difficult to obtained. PS used in the paper was 
derived from soybeans and no obvious adverse reactions occurred after continuous administration for 20 weeks. Moreover, the memory of AD patients significantly improved. Thus, the safety and effectiveness of short-term PS treatment was confirmed. However, AD patients require lifelong medication and the safety and effectiveness of PS over the long-term has not yet been proven. Second, there were no abnormalities around the blood vessels and white matter in rats receiving either PS doses of 15 or $30 \mathrm{mg} / \mathrm{kg}$. However, there were calcifications around the blood vessels and/or white matter in three rats in the AD model group. The pathological properties of brain atrophy in AD patients is different from white matter calcification, and a correlation between calcification in the brain and AD has not been found. Therefore, whether these lesions are associated with $\mathrm{AD}$, memory reduction in $\mathrm{AD}$, or just spontaneous lesions, is unknown, and requires further investigation.

In conclusion, the application of PS can improve memory function in AD patients, reduce the levels of brain cholinesterase, improve SOD and hydroxyl free radical inhibition rate, and improve hippocampal inflammatory. However, the underlying mechanisms of these actions require further investigation.

\section{ACKNOWLEDGMENTS}

Research supported by Qigihar Philosophy and Social Science Research Project (\#QSX2014--30QN).

\section{REFERENCES}

Ballard C, Gauthier S, Corbett A, Brayne C, et al. (2011). Alzheimer's disease. Lancet 377: 1019-1031.

Cagnin A, Cester A, Costa B, Ermani M, et al. (2014). Effectiveness of switching to the rivastigmine transdermal patch from oral cholinesterase inhibitors: a naturalistic prospective study in Alzheimer's disease. Neurol. Sci. 36: 457-463.

Canale C, Seghezza S, Vilasi S, Carrotta R, et al. (2013). Different effects of Alzheimer's peptide A $\beta(1-40)$ oligomers and fibrils on supported lipid membranes. Biophys. Chem. 182: 23-29.

Cong L and Jia J (2011). Promoter polymorphisms which regulate ADAM9 transcription protect against sporadic Aizheimer's disease. Neurobiol. Aging 32: 54-62.

Grimm MO, Haupenthal VJ, Rothhaar TL, Zimmer VC, et al. (2013). Effect of different phospholipids on alpha-secretase activity in the non-amyloidogenic pathway of Alzheimer's disease. Int. J. Mol. Sci. 14: 5879-5898.

Hirayama S, Terasawa K, Rabeler R, Hirayama T, et al. (2014). The effect of phosphatidylserine administration on memory and symptoms of attention-deficit hyperactivity disorder: a randomised, double-blind, placebo-controlled clinical trial. J. Hum. Nutr. Diet (Suppl 2): 284-291.

Hou Y, Wang Y, Zhao J, Li X, et al. (2014). Smart soup, a traditional Chinese medicine formula, ameliorates amyloid pathology and related cognitive deficits. PLoS One 9: e111215.

Izzo NJ, Xu J, Zeng C, Kirk MJ, et al. (2014). Alzheimer's therapeutics targeting amyloid beta 1-42 oligomers II:Sigma-2/ PGRMC1 receptors mediate abeta 42 oligomer binding and synaptotoxicity. PLoS One 9: e111899.

Liu SH, Chang CD, Chen PH, Su JR, et al. (2012). Docosahexaenoic acid and phosphatidylserine supplementations improve antioxidant activities and cognitive functions of the developing brain on pentylenetetrazol-induced seizure model. Brain Res. 1451: 19-26.

Maiese K and Chong ZZ (2004). Lights into oxidative stress and potential novel therapeutic targets for Alzheimer disease. Restor. Neurol. Neurosci. 22: 87-104.

Manor I, Magen A, Keidar D, Rosen S, et al. (2012). The effect of phosphatidylserine containing Omega3 fatty-acids on attention-deficit hyperactivity disorder symptoms in children: a double-blind placebo-controlled trial, followed by an open-label extension. Eur. Psychiatr. 27: 335-342.

Meng X, Wang M, Sun G, Ye J, et al. (2014). Attenuation of A $325-35$-induced parallel autophagic and apoptotic cell death by gypenoside XVII through the estrogen receptor-dependent activation of Nrf2/ARE pathways. Toxicol. Appl. Pharmacol. 279: 63-75. 
Nair S, Traini M, Dawes IW and Perrone GG (2014). Genome-wide analysis of Saccharomyces cerevisiae identifies cellular processes affecting intracellular aggregation of Alzheimer's amyloid- $\beta 42$ : importance of lipid homeostasis. Mol. Biol. Cell 25: 2235-22349.

Penolazzi B, Bergamaschi S, Pastore M, Villani D, et al. (2014). Transcranial direct current stimulation and cognitive training in the rehabilitation of Alzheimer disease: A case study. Neuropsychol. Rehabil. 7: 1-19.

Richter Y, Herzog Y, Lifshitz Y, Hayun R, et al. (2013). The effect of soybean-derived phosphatidylserine on cognitive performance in elderly with subjective memory complaints: a pilot study. Clin. Interv. Aging 8: 557-563.

Sato Y, Nakamura T, Aoshima K and Oda Y (2010). Quantitative and wide-ranging profiling of phospholipids in human plasma by two-dimensional liquid chromatography/mass spectrometry. Anal. Chem. 82: 9858-9864.

Schmid D, Grabmer C, Streif D, Lener T, et al. (2014). T-Cell death, phosphatidylserine exposure and reduced proliferation rate to validate extracorporeal photochemotherapy. Vox Sang. 108: 82-88.

Suzuki S, Yamatoya H, Sakai M, Kataoka A, et al. (2001). Oral administration of soybean lecithin transphosphatidylated phosphatidylserine improves memory impairment in aged rats. J. Nutr. 131: 2951-2956.

Wollen KA (2010). Alzheimer's disease: the pros and cons of pharmaceutical, nutritional, botanical, and stimulatory therapies, with a discussion of treatment strategies from the perspective of patients and practitioners. Altern. Med. Rev 15: 223-424.

Zanotta D, Puricelli S and Bonoldi G (2014). Cognitive effects of a dietary supplement made from extract of Bacopa monnieri, astaxanthin, phosphatidylserine, and vitamin $\mathrm{E}$ in subjects with mild cognitive impairment: a noncomparative, exploratory clinical study. Neuropsychiatr. Dis. Treat 10: 225-230. 